\title{
Hypercalcemia
}

\section{Sophy $\mathrm{Mo}^{1}$}

${ }^{1}$ Faculty of Medicine, McGill University

Correspondence

Sophy Mo

Email: sophy.mo@mail.mcgill.ca

Publication Date

January 8, 2022

MJM 2022 (20) 19

https://doi.org/10.26443/mjm.v20i1.941

\section{\% McGill \\ Journal of Medicine}

www.mjmmed.com

\section{(c) (i) (2)(2)}

This work is licensed under a Creative Commons BY-NC-SA 4.0 International License.

\section{ABSTRACT}

Hypercalcemia is a presentation commonly encountered in the clinical setting. Due to its vast differential diagnosis, a systematic approach is necessary when approaching patients with hypercalcemia. This article presents a simple, yet thorough approach to help clinicians determine the etiology of their patients' hypercalcemia. The main components of history taking, physical examination, and laboratory investigations for patients with hypercalcemia are highlighted. Emphasis is put on the importance of determining whether the hypercalcemia is associated with elevated or inappropriately normal parathyroid hormone (PTH) levels or not. The main etiologies of PTH-dependent hypercalcemia and PTHindependent hypercalcemia are explored. Primary hyperparathyroidism and hypercalcemia secondary to malignancy are highlighted as together, they make up $90 \%$ of hypercalcemia cases. A presentation of the management principles of hypercalcemia is also provided.

\section{KEYWORDS}

Ionized calcium, Total calcium, Malignancy-associated hypercalcemia, Hyperparathyroidism, Hypercalcemia.

\section{1 | QUESTION}

A 57-year-old female presents to your family medicine clinic for the first time after being told her calcium levels were elevated $(2.78 \mathrm{mmol} / \mathrm{L})$ at a recent emergency room visit for a migraine headache. No other abnormalities were noted on her complete blood count, electrolytes, and renal function tests at the time. She is known for migraines, osteoarthritis, hypertension, and dyslipidemia. Her medications are atorvastatin $20 \mathrm{mg}$ PO DIE and amlodipine $5 \mathrm{mg}$ PO DIE. On history, she describes feeling well at the time of her visit and denies having any family members with calcium abnormalities.
She reports occasional bilateral knee pain, which she attributes to her osteoarthritis. Her vital signs are stable and her physical exam is unremarkable.

Among the following, which would you send as part of your initial investigations for this patient?
A. Serum protein electrophoresis (SPEP)
B. Parathyroid hormone (PTH)
C. 24-hour urine collection for calcium and creatinine
D. PTH-related peptide (PTHrP)
E. Chest radiograph 


\section{2 | ANSWER}

B. Initial investigations for hypercalcemia should include a repeat total and ionized calcium to confirm the diagnosis of hypercalcemia. Albumin levels should also be ordered since abnormally low albumin levels may affect the total serum calcium level measured. Most physicians will also order extended electrolytes, renal function tests, and a complete blood count. One of the most important initial investigations is the serum PTH level as depending on whether it is normal/high or low, the differential diagnoses are very different. If the $\mathrm{PTH}$ is low, PTHrP (D), SPEP (A), and a chest radiograph (E) may be sent as part of a malignancy workup. If the PTH is normal or high, a 24-hour urine collection for calcium and creatinine $(\mathrm{C})$ is often ordered to assess for familial hypocalciuric hypercalcemia $(\mathrm{FHH})$. However, these would not be done prior to ordering a PTH.

\section{3 | INITIAL APPROACH}

\section{1 | Clinical Assessment}

During history taking of a patient with hypercalcemia, the focus should be on eliciting the signs and symptoms of hypercalcemia as well as its possible etiologies. The clinical manifestations of hypercalcemia can be remembered by the mnemonic "stones, bones, abdominal moans, and psychic groans", which refers to its renal, skeletal, gastrointestinal, and neuromuscular manifesta- tions. Psychiatric and cardiovascular manifestations can also occur. These are detailed in Table 1. It is important to remember that patients with mild hypercalcemia, defined as values not exceeding $0.25 \mathrm{mmol} / \mathrm{L}$ above normal range or $<3 \mathrm{mmol} / \mathrm{L}$, may be asymptomatic (1-3).

Since hypercalcemia may be due to multiple etiologies, performing a thorough review of medications, family history, and past medical history is essential. For example, clinicians should review the patient's medications for drugs that could cause hypercalcemia. Moreover, a family history of hypercalcemia might suggest a genetic mutation resulting in hypercalcemia (1). Clinicians should also inquire about clinical features suggestive of various cancers as well as the patient's most recent cancer screenings since malignancy is a known etiology of hypercalcemia (2). Lastly, the patient's past medical history can reveal medical conditions associated with hypercalcemia such as endocrine disorders, granulomatous diseases, or kidney disease (2).

\section{2 | Initial Laboratory Investigations}

Hypercalcemia is defined as a serum calcium level greater than two standard deviations above the normal mean in a reference laboratory. Hypercalcemia should be confirmed with repeat measurements of ionized cal-

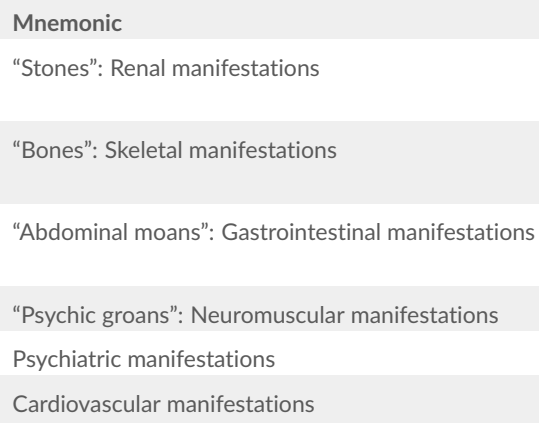

TAB LE 1 Clinical manifestations of hypercalcemia.

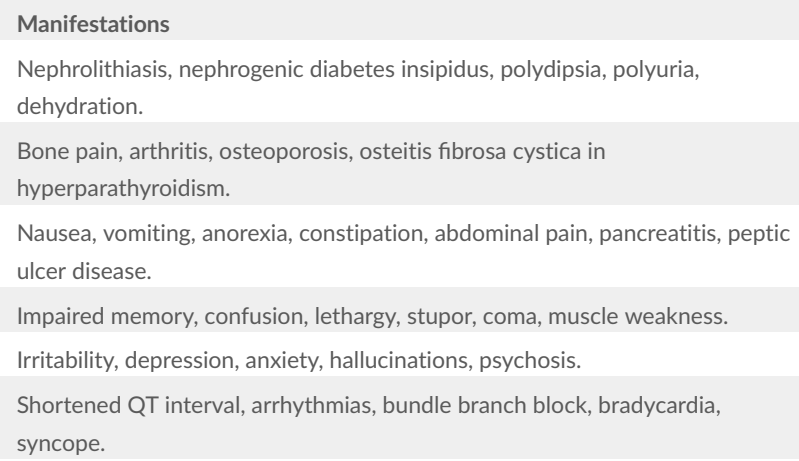

Nausea, vomiting, anorexia, constipation, abdominal pain, pancreatitis, peptic ulcer disease.

Impaired memory, confusion, lethargy, stupor, coma, muscle weakness. Irritability, depression, anxiety, hallucinations, psychosis.

Shortened QT interval, arrhythmias, bundle branch block, bradycardia, syncope. 
cium and total calcium. However, it is important to account for biologic and analytic variation when interpreting repeat measurements to determine if one value is truly different from the other and if a value is significantly elevated (4). Although the reference values can vary depending on the laboratory, normal total calcium generally ranges between $2.15-2.60 \mathrm{mmol} / \mathrm{L}$ and ionized calcium usually ranges between 1.17-1.33 mmol/L (1). Low albumin levels may also affect total serum calcium level (1). However, the single most important investigation for hypercalcemia is the PTH level. Hence, we will divide the different diagnoses for hypercalcemia depending on if the PTH is normal or high (PTH-dependent hypercalcemia) or if the PTH is low (PTH-independent hypercalcemia). Although the causes of hypercalcemia are numerous, $90 \%$ of all cases of hypercalcemia result from primary hyperparathyroidism or malignancy (1).

\section{3 | PTH-Dependent Hypercalcemia}

In PTH-dependent hypercalcemia, PTH is inappropriately normal or high despite elevated calcium levels. The most common cause is primary hyperparathyroidism. $80 \%$ of these cases result from a single parathyroid adenoma. It can also be associated with hyperplasia of the parathyroid glands. Often, patients presenting with mild asymptomatic hypercalcemia will be found to have primary hyperparathyroidism (1).

Primary hyperparathyroidism can be distinguished from familial hypocalciuric hypercalcemia $(\mathrm{FHH})$ by performing a 24-hour urine collection for calcium and creatinine. $\mathrm{FHH}$ is associated with a low calcium to creatinine clearance ratio $(<0.01)$ whereas primary hyperparathyroidism would lead to a high ratio ( $>0.02)$. For practical purposes, urinary calcium to creatinine ratio or calcium concentration in a spot urine sample is often used for the initial evaluation of calciuria. If clinically indicated, calciuria can be confirmed with a 24-hour urine collection. $\mathrm{FHH}$ is an autosomal dominant condition caused by a calcium-sensing receptor gene mutation. It is important to distinguish between $\mathrm{FHH}$ and primary hyperparathyroidism as the management differs significantly. For example, parathyroidectomy is not appro- priate for $\mathrm{FHH}$ (5). However, $\mathrm{FHH}$ is rare and usually benign. Hence, when faced with PTH-dependent hypercalcemia in clinical practice, physicians will often focus on ordering imaging studies of the parathyroid to assess for adenoma rather than the 24-hour urine collection.

Moreover, primary hyperparathyroidism may be a presentation of multiple endocrine neoplasia type 1 (MEN 1) syndrome, where parathyroid hyperplasia or adenoma occurs with pituitary and pancreatic islet tumors. It is also associated with MEN 2A syndrome, which is characterized by medullary thyroid carcinoma, pheochromocytoma, and parathyroid hyperplasia or adenoma. Hence, it is important to inquire about these associated conditions in such patients as well as perform a thorough family history. As these syndromes are hereditary, genetic testing is usually performed if there is a high clinical suspicion (5).

Other causes of PTH-dependent hypercalcemia include tertiary hyperparathyroidism. It occurs in patients with end-stage renal disease resulting in prolonged secondary hyperparathyroidism, high phosphate, and low vitamin D. Over time, this leads to autonomous PTH secretion unresponsive to plasma calcium levels. The drug lithium can also cause PTH-dependent hypercalcemia, which may be reversed if the drug is discontinued (3).

\section{4 | PTH-Independent Hypercalcemia}

In PTH-independent hypercalcemia, PTH is appropriately suppressed from high calcium levels.

Malignancy: Malignancy is a common etiology of hypercalcemia and can result from various mechanisms: osteolysis from metastases, excess PTH-related peptide (PTHrP), excess calcitriol production, and multiple myeloma (6). Hence, many clinicians will pursue investigations to search for an occult malignancy in PTH-independent hypercalcemia. PTHrP, alkaline phosphatase, calcitriol, and multiple myeloma workup are usually included as part of the laboratory investigations. In terms of imaging, a mammogram, chest radiograph, and abdominal CT are often done (2).

Osteolysis from metastasis: Bone metastases resulting in extensive osteolysis can result in hypercalcemia. 
The most frequently associated malignancies are multiple myeloma and breast cancer. A clue to this etiology of malignancy-associated hypercalcemia is a high alkaline phosphatase level. Lytic lesions may also be seen on imaging (3).

Excess PTHrP: Some solid tumors secrete PTHrP. Although PTH is suppressed, PTHrP acts similarly to PTH by increasing bone resorption and calcium reabsorption in the kidney. It is most commonly associated with squamous cell carcinomas of the lung, head and neck, esophagus, skin, or cervix, and carcinomas of the breast, kidney, prostate, and bladder (7).

Excess calcitriol (1,25 dihydroxyvitamin D): Lymphomas may cause increased production of calcitriol as a result of their $1 \alpha$-hydroxylase activity. The excess calcitriol subsequently leads to increased renal and gastrointestinal absorption of calcium. Hence, lymphoma patients may present with hypercalcemia (6).

Multiple myeloma: Multiple myeloma can also result in hypercalcemia due to bone lysis. It can be suspected if there is presence of other typical signs and symptoms of the disease: anemia, renal insufficiency, and bone pain. Serum and urine protein electrophoresis and serum free light chains are commonly ordered to investigate for multiple myeloma. Lytic lesions in the bone may also be seen on skeletal survey (8).

No evidence of malignancy: If no evidence of malignancy is found, other less common causes of PTHindependent hypercalcemia can be considered. These can be divided into the following categories: endocrinopathies, granulomatous diseases, drugs, and immobilization (1).

Endocrinopathies: Hypercalcemia can be associated with hyperthyroidism due to increased bone resorption (3). Although less common, adrenal insufficiency has also been reported as a cause of hypercalcemia (9). Although the mechanism of hypercalcemia in adrenal insufficiency is not entirely clear, it is thought that the hypovolemia associated with adrenal insufficiency results in decreased glomerular filtration rate. Hence, the amount of calcium filtered at the glomerulus is also decreased (9).

Granulomatous diseases: In granulomatous diseases, excess calcitriol is synthesized in macrophages and other cells in granulomas, which disturbs the usual production of calcitriol regulated by calcium, phosphate, and PTH levels. Consequently, it may result in hypercalcemia (5). Examples of granulomatous diseases include sarcoidosis, berylliosis, tuberculosis, fungal infections, and Crohn's disease (10).

Drugs: Thiazide diuretics are a common cause of drugrelated hypercalcemia as they increase renal reabsorption of calcium. Around $8 \%$ of patients taking thiazides develop hypercalcemia (11). Moreover, ingestion of large amounts of calcium or calcium-containing antacids may result in milk-alkali syndrome, which is characterized by hypercalcemia, alkalosis, and renal failure (5). Excessive ingestion of Vitamin A and Vitamin D may also lead to hypercalcemia. Vitamin $A$ is thought to stimulate osteoclastic resorption and inhibit osteoblastic formation (1).

Immobilization: Immobilization may rarely result in hypercalcemia due to decreased bone formation and increased bone resorption. This usually occurs in individuals with a high bone turnover such as in Paget's disease or in younger individuals (5).

\section{4 | BEYOND INITIAL APPROACH}

The management of hypercalcemia will depend on the underlying etiology. Mild hypercalcemia is defined as values not exceeding $0.25 \mathrm{mmol} / \mathrm{L}$ above normal range or $<3 \mathrm{mmol} / \mathrm{L}$ (1). Most instances of mild hypercalcemia are from primary hyperparathyroidism. If it does not result in any symptoms (e.g., skeletal, renal, gastrointestinal, or neuromuscular), patients can usually be monitored without surgery (3). Indications for parathyroidectomy in primary hyperparathyroidism include overt clinical manifestations, calcium $>0.25 \mathrm{mmol} / \mathrm{L}$ above normal, creatinine clearance $<60 \mathrm{~mL} / \mathrm{min}$, low bone mineral density, history of fragility fracture, and age $<50$ years old (2). The management of hypercalcemia from other etiologies is beyond the scope of the current article.

Acute, severe hypercalcemia is defined as serum calcium greater than $3.5 \mathrm{mmol} / \mathrm{L}$. However, indications for urgent treatment of hypercalcemia are more based on the clinical manifestations than the serum level. The 
mainstay of management consists of aggressive intravenous fluids and intravenous bisphosphonate. Calcitonin is sometimes used as a temporizing measure. Furosemide, glucocorticoids, and dialysis can also be indicated in some patients (2).

In rare cases, severe hypercalcemia may result in confusion and decreased level of consciousness. Airway management is crucial in the care of such patients. It may also result in hemodynamic instability requiring fluid resuscitation. Moreover, it can induce arrhythmias. Hence, these patients should undergo an electrocardiogram. Hemodialysis against a low or zero calcium dialysate should be considered in these potentially life-threatening cases when significant cardiovascular or neurologic dysfunction is present (1).

\section{REFERENCES}

1. Minisola S, Pepe J, Piemonte S, Cipriani C. The diagnosis and management of hypercalcaemia. BMJ : British Medical Journal. 2015;350:h2723. doi: 10.1136/bmj.h2723

2. Melmed S, Williams RH. Williams textbook of endocrinology. 12th ed. Philadelphia: Elsevier/Saunders; 2011.

3. Carroll MF, Schade DS. A practical approach to hypercalcemia. American family physician. 2003;67(9):1959-66.

4. McCormack JP, Holmes DT. Your results may vary: the imprecision of medical measurements. BMJ. 2020;368:m149.

5. Jameson JL, Kasper DL, Longo DL, Fauci AS, Hauser SL, Loscalzo J. Harrison's principles of internal medicine. 20th edition. ed. New York: McGraw-Hill Education; 2018.

6. Reagan P, Pani A, Rosner MH. Approach to diagnosis and treatment of hypercalcemia in a patient with malignancy. American journal of kidney diseases : the official journal of the National Kidney Foundation. 2014;63(1):141-7. doi: 10.1053/j.ajkd.2013.06.025

7. Zagzag J, Hu MI, Fisher SB, Perrier ND. Hypercalcemia and cancer: Differential diagnosis and treatment. CA Cancer J Clin. 2018;68(5):377-86. doi: 10.3322/caac.21489

8. Nau KC, Lewis WD. Multiple myeloma: diagnosis and treatment. Am Fam Physician. 2008;78(7):853-9.

9. Ahn SW, Kim TY, Lee S, Jeong JY, Shim H, Han YM, et al. Adrenal insufficiency presenting as hypercalcemia and acute kidney injury. Int Med Case Rep J. 2016;9:223-6. doi: 10.2147/IMCRJ.S109840 10. Sharma OP. Hypercalcemia in granulomatous disorders: a clinical review. Current opinion in pulmonary medicine. 2000;6(5):4427. doi: 10.1097/00063198-200009000-00010

11. Wermers RA, Kearns AE, Jenkins GD, Melton LJ, 3rd. Incidence and clinical spectrum of thiazide-associated hypercalcemia. Am J Med. 2007;120(10):911.e9-15. doi: 10.1016/j.amjmed.2006.07.044

12. Toronto Notes 2020: Comprehensive Medical Reference and Review for the Medical Council of Canada Qualifying Exam (MCCQE) Part I and the United States Medical Licensing Exam (USMLE) Step II: Toronto Notes for Medical Students, Incorporated; 2020. 


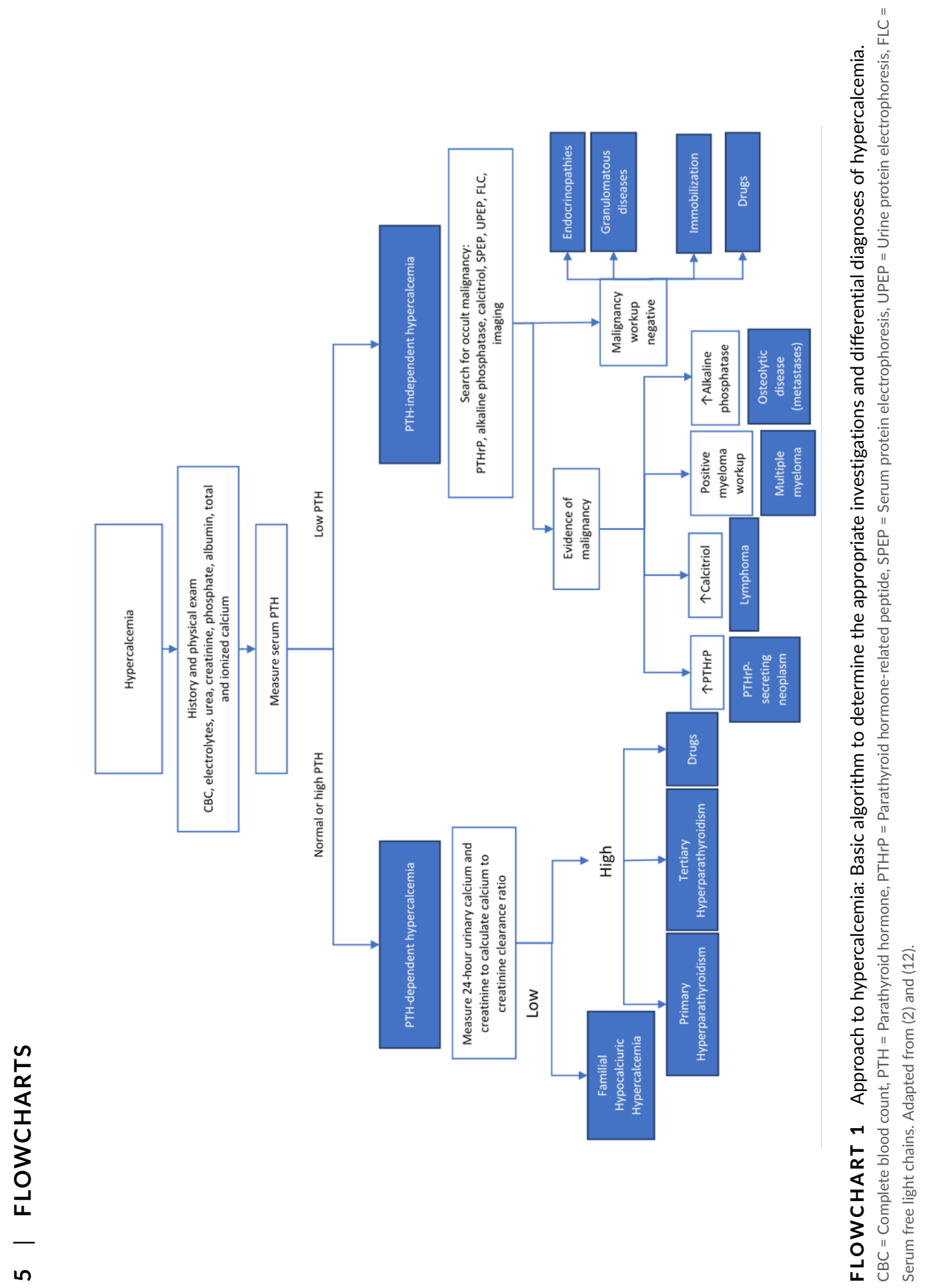

